\title{
Neural Network-Based Adaptive Backstepping Control for Hypersonic Flight Vehicles with Prescribed Tracking Performance
}

\author{
Zhu Guoqiang and Liu Jinkun \\ School of Automation Science and Electrical Engineering, Beihang University, Beijing 100191, China \\ Correspondence should be addressed to Liu Jinkun; ljk@buaa.edu.cn
}

Received 1 December 2014; Revised 11 April 2015; Accepted 15 April 2015

Academic Editor: Hak-Keung Lam

Copyright (C) 2015 Z. Guoqiang and L. Jinkun. This is an open access article distributed under the Creative Commons Attribution License, which permits unrestricted use, distribution, and reproduction in any medium, provided the original work is properly cited.

An adaptive neural control scheme is proposed for a class of generic hypersonic flight vehicles. The main advantages of the proposed scheme include the following: (1) a new constraint variable is defined to generate the virtual control that forces the tracking error to fall within prescribed boundaries; (2) RBF NNs are employed to compensate for complex and uncertain terms to solve the problem of controller complexity; (3) only one parameter needs to be updated online at each design step, which significantly reduces the computational burden. It is proved that all signals of the closed-loop system are uniformly ultimately bounded. Simulation results are presented to illustrate the effectiveness of the proposed scheme.

\section{Introduction}

During the past decades, hypersonic flight vehicles (HFVs) have received a great deal of attention. They may represent more cost-efficient and reliable access to space routine and are especially suitable for prompt global response, as well as offering worldwide air superiority because of the high speed and endurance [1-5]. In this paper a nonlinear generic model of HFVs is adopted, which has been widely used by various researchers [6-8]. The dynamics of HFVs are highly nonlinear with strong couplings between the propulsive and aerodynamic effects. The requirements of flight stability and high speed response make the onboard flight control of HFVs quite difficult $[9,10]$. Besides, modeling inaccuracy can result in strong adverse effects on the performance of HFVs control systems. Thus, the controller design for HFVs is challenging and must guarantee closed-loop stability and desired performance [11].

Recently, feedback control strategy based on nonlinear control theory has been used for HFVs, such as sliding mode control [3], minimax linear quadratic regulator control $[12,13]$, genetic algorithm [14], and sequential loop closure controller design [15]. In [16], the adaptive backstepping method was used to design controller for the HFVs model, while fuzzy logic and neural networks were used to approximate the unknown system dynamics in [17-19]. Adaptive dynamic surface control schemes were proposed by $[20,21]$ to avoid the derivatives of nonlinear functions. The nonlinear dynamic inversion method was used to design a robust controller. In $[3,14]$, feedback linearization techniques were applied to design nonlinear controllers for the longitudinal motion of a hypersonic aircraft containing aerodynamic uncertain parameters. This approach leads to a complicated high-order Lie derivatives and is hard to perform a robustness analysis when considering uncertainties. In [22], a neural network controller for a nonlinear flight dynamic system was designed by using the adaptation mechanism to deal with the effects of aerodynamic modeling errors.

In the control design for HFVs, an important issue is tracking performance. Traditionally, the controller for HFVs guarantees the tracking error convergence to a residual set. Moreover, the transient behavior such as overshoot, undershoot, and convergence rate are difficult to be established analytically. In [23-25], a prescribed performance scheme is proposed for one-class nonlinear systems; this approach is to construct a prescribed performance function that converts 
the tracking error into a new variable. Therefore the tracking performance can be characterized by a prescribed constraint function. Besides, the prescribed performance approach with new definition is applied in a class of uncertain strictfeedback systems [26], strict-feedback time-delay systems [27], and MIMO systems [28], respectively.

A drawback of adaptive NNs [22] or FLSs $[29,30]$ schemes is that the number of adaptation laws generally depends on the neural network nodes or the fuzzy rules. That is, with an increase of the nodes or the rules, the parameters to be estimated may be greatly increased. To solve this problem, we propose a new method by estimating the norm of the NNs weights rather than estimating every item of the weight vector [31-33].

In this paper, we separate the longitudinal model of HFVs into two parts: the velocity subsystem and the altitude subsystem. Velocity and altitude controllers are designed separately. For the velocity subsystem, a dynamic inversion controller with radial basis function neural networks (RBF $\mathrm{NNs}$ ) is proposed to track a desired velocity trajectory. The altitude subsystem is transformed into a strict-feedback form. Then an adaptive backstepping controller is designed to track a desired altitude trajectory. The main contribution of this paper is described as follows:

(1) We introduce a performance function, and a new error constraint variable is used as a virtual tracking error variable to ensure the prescribed transient performance. By extending the prescribed tracking performance technique proposed in $[23,24]$ to HFVs, it is shown that the tracking errors can converge to predefined arbitrarily small residue sets with prescribed convergence rate and maximum overshoot.

(2) RBF NNs are employed to compensate for complex and uncertain terms to solve the problem of controller complexity. By using the minimal learning technique [31-33], only one parameter needs to be updated online at each design step regardless of the NNs inputoutput dimension and the number of NNs nodes. As a result, the number of adaptation laws, which generally depends on the neural network nodes, and the computational burden are greatly reduced.

(3) With the bounded of the virtual control gain $g_{i}(\cdot)$, the singularity problem by the estimation of $g_{i}(\cdot)$ is avoided without any effort, and both low and up bounded will not appear in the control law and will be used only for analysis; they can be unknown.

The rest of this paper is organized as follows. In Section 2, the nonlinear longitudinal dynamic model of HFVs is presented. The controllers design and the stability analysis are given in Section 3. The simulation results are illustrated in Section 4, followed by conclusions of this paper in Section 5 .

\section{Problem Formulation and Preliminaries}

2.1. Longitudinal Model of HFVs. The model considered in this paper is taken from the NASA Langley Research Center
$[2,3]$. Cruising at a Mach number of 15 and at an altitude of $110000 \mathrm{ft}$, the longitudinal hypersonic flight model is given by

$$
\begin{aligned}
& \dot{V}=\frac{T(V, \beta) \cos \alpha-D(V, \alpha)}{m}-\frac{\mu \sin \gamma}{r^{2}}, \\
& \dot{h}=V \sin \gamma, \\
& \dot{\gamma}=\frac{L(V, \alpha)+T(V, \beta) \sin \alpha}{m V}-\frac{\left(\mu-V^{2} r\right) \cos \gamma}{V r^{2}}, \\
& \dot{\alpha}=q-\dot{\gamma}, \\
& \dot{q}=\frac{M_{y y}\left(V, \alpha, q, \delta_{E}\right)}{I_{y y}},
\end{aligned}
$$

where $V$ is the velocity, $\gamma$ the flight path angle, $h$ the altitude, $\alpha$ the attack angle, $q$ the pitch rate, $\delta_{E}$ the elevator deflection, and $\beta$ the throttle setting. $T(V, \beta), D(V, \alpha), L(V, \alpha)$, and $M_{y y}\left(V, \alpha, q, \delta_{E}\right)$ represent the thrust, drag, lift-force, and pitching moment, respectively, which can be expressed as

$$
\begin{aligned}
T(V, \beta) & =\frac{\rho V^{2} S C_{T}}{2}, \\
L(V, \alpha) & =\frac{\rho V^{2} S C_{L}}{2}, \\
D(V, \alpha) & =\frac{\rho V^{2} S C_{D}}{2}, \\
M_{y y} & =\frac{1}{2} \rho V^{2} S \bar{c}\left[C_{M}(\alpha)+C_{M}\left(\delta_{E}\right)+C_{M}(q)\right],
\end{aligned}
$$

with

$$
\begin{aligned}
C_{L}= & 0.6203 \alpha, \\
C_{D}= & 0.6450 \alpha^{2}+0.0043378 \alpha+0.003772, \\
C_{T}= & \begin{cases}0.02576 \beta & \text { if } \beta<1 \\
0.0224+0.00336 \beta & \text { if } \beta>1,\end{cases} \\
r= & h+R_{E}, \\
C_{M}(\alpha)= & -0.035 \alpha^{2}+0.036617\left(1+\Delta C_{M \alpha}\right) \alpha \\
& +5.3261 \times 10^{-6}, \\
C_{M}(q)= & \left(\frac{c}{2 V}\right) q\left(-6.796 \alpha^{2}+0.3015 \alpha-0.2289\right), \\
C_{M}\left(\delta_{E}\right)= & c_{e}\left(\delta_{E}-\alpha\right) .
\end{aligned}
$$

The nominal values of inertial and aerodynamic parameters are given in Table 1. Besides, at trimmed cruise condition, $V=15060 \mathrm{ft} / \mathrm{s}, h=11000 \mathrm{ft}, \gamma=0 \mathrm{rad}, \alpha=0.0315$, and $q=0 \mathrm{rad} / \mathrm{s}$.

The engine dynamics can be modeled by a second-order system:

$$
\ddot{\beta}=-2 \xi \omega_{n} \dot{\beta}-\omega_{n}^{2} \beta+\omega_{n}^{2} \beta_{c} .
$$


TABLE 1: Parameters of the HFV.

\begin{tabular}{llc}
\hline Symbol & Parameter & Value \\
\hline $\bar{c}$ & Mean aerodynamic chord & $80 \mathrm{ft}$ \\
$I_{y y}$ & Moment of inertia & $7 \times 10^{6} \mathrm{slug} \cdot \mathrm{ft}^{2}$ \\
$S$ & Reference area & $3603 \mathrm{ft}^{2}$ \\
$\rho$ & Air density & $0.24325 \times 10^{-4} \mathrm{slugs} \cdot \mathrm{ft}^{-3}$ \\
$m$ & Mass of aircraft & $9375 \mathrm{slug}$ \\
$R_{E}$ & Radius of the earth & $20903500 \mathrm{ft}$ \\
$\mu$ & Gravitational constant & $1.39 \times 10^{16} \mathrm{ft}^{3} / \mathrm{s}^{2}$ \\
\hline
\end{tabular}

Therefore, by selecting the commanded value $\beta_{c}$ as the new control input, the HFV is composed of five state variables $X=[V, h, \gamma, \alpha, q]^{T}$ and two control inputs $U=\left[\beta_{c}, \delta_{E}\right]^{T}$, while the outputs to be controlled are selected as $Y=$ $[V, h]^{T}$. The design objective is such that the outputs track the desired altitude and velocity commands $Y_{d}=\left[V_{d}, h_{d}\right]^{T}$ with prescribed tracking performance.

From (1), it can be inferred that the main contribution in the change of flight vehicle velocity is from the throttle setting $\beta_{c}$. The altitude change is related mainly to the elevator deflection $\delta_{E}$. Thus, it is reasonable to divide the system into two loops: the velocity loop and the altitude loop.

Note that the thrust term $T \sin \alpha$ is generally much smaller than the lift $L$, velocity $V$ is high, and the flight path angle $\gamma$ is typically very small during the trimmed cruise condition, which justify the following approximation.

Assumption 1 (see $[7,19]$ ). The thrust term $T \sin \alpha \approx 0$, and the term $V \sin \gamma \approx V \gamma$.

Defining that $\theta$ denotes the pitch angle, we have $\theta=\alpha+\gamma$. Then, we define state variables as $x=\left[x_{1}, x_{2}, x_{3}, x_{4}\right]^{T}$, with $x_{1}=h, x_{2}=\gamma, x_{3}=\theta$, and $x_{4}=q$. For simplicity, let $\bar{x}_{i}=$ $\left[x_{1}, \ldots, x_{i}\right]^{T}$, so the altitude subsystem can be written as

$$
\begin{aligned}
& \dot{x}_{1}=f_{1}\left(\bar{x}_{1}\right)+g_{1}\left(\bar{x}_{1}\right) x_{2}, \\
& \dot{x}_{2}=f_{2}\left(\bar{x}_{2}\right)+g_{2}\left(\bar{x}_{2}\right) x_{3}, \\
& \dot{x}_{3}=f_{3}\left(\bar{x}_{3}\right)+g_{3}\left(\bar{x}_{3}\right) x_{4}, \\
& \dot{x}_{4}=f_{4}\left(\bar{x}_{4}\right)+g_{4}\left(\bar{x}_{4}\right) \delta_{E}, \\
& y=x_{1},
\end{aligned}
$$

where

$$
\begin{aligned}
& f_{1}\left(\bar{x}_{1}\right)=0 \\
& f_{3}\left(\bar{x}_{3}\right)=0 \\
& f_{2}\left(\bar{x}_{2}\right)=-\frac{\rho V^{2} S}{2 m V} 0.6203 x_{2}-\frac{\left(\mu-V^{2} r\right)}{V r^{2}} \cos x_{2}, \\
& f_{4}\left(\bar{x}_{4}\right)=\frac{\bar{c} \rho V^{2} S}{2 I_{y y}}\left(C_{M}\left(x_{3}-x_{2}\right)+C_{M}\left(x_{4}\right)\right. \\
& \left.-0.0292\left(x_{3}-x_{2}\right)\right), \\
& g_{1}\left(\bar{x}_{1}\right)=V,
\end{aligned}
$$

$$
\begin{aligned}
& g_{2}\left(\bar{x}_{2}\right)=\frac{\rho V^{2} S}{2 m V} 0.6203 \\
& g_{3}\left(\bar{x}_{3}\right)=1 \\
& g_{4}\left(\bar{x}_{4}\right)=\frac{c_{e} \bar{c} \rho V^{2} S}{2 I_{y y}}
\end{aligned}
$$

Since the values of the inertial and the aerodynamic parameters are uncertain, the aforementioned $f_{i}\left(\bar{x}_{i}\right)$ and $g_{i}\left(\bar{x}_{i}\right), i=1,2,3,4$, are unknown smooth functions. Moreover, it is easy to check that $g_{i}\left(\bar{x}_{i}\right)$ are always strictly positive. With these observations in mind, we have the following assumption.

Assumption 2. There exist positive constants $b_{i}$ and $d_{i}$ such that $0<b_{i} \leq\left|g_{i}\left(\bar{x}_{i}\right)\right| \leq d_{i}$.

Remark 3. It is worth noting that, in the proposed scheme, both $b_{i}$ and $d_{i}$ will not appear in the control law and will be used only for analysis; they can be unknown.

Assumption 4. $V_{d}$ and its first derivative are known and bounded, while $h_{d}$ and its first four derivatives are continuous and bounded.

2.2. Description of RBF NNs. In this paper, RBF NNs will be employed to approximate unknown functions. Mathematically, an RBF NN can be expressed as

$$
F(\xi)=W^{T} \psi(\xi),
$$

where $F \in \mathbb{R}$ and $\xi \in \mathbb{R}^{n}$ are the NN outputs and input, $W \in \mathbb{R}^{n}$ is the weight vector, and $\psi(\xi)=\left[\psi_{1}(\xi), \ldots, \psi_{N}(\xi)\right]^{T}$ is the basis function vector with $\psi_{i}(\xi)$ commonly chosen as the Gaussian functions:

$$
\begin{aligned}
\psi_{i}(\xi)=\frac{1}{\sqrt{2 \pi} \phi} \exp \left(-\frac{\left\|\xi-\xi_{i}\right\|^{2}}{2 \phi^{2}}\right), & \\
& \phi>0, i=0, \ldots, N,
\end{aligned}
$$

where $\xi_{i} \in \mathbb{R}^{n}$ and $\phi \in \mathbb{R}$ are constants called the center and width of the basis function, respectively.

Lemma 5 (see [17]). Given any continuous function $F(\xi)$ : $\Omega_{\xi} \rightarrow \mathbb{R}$ with $\Omega_{\xi} \subset \mathbb{R}^{n}$ a compact set and any constant $\epsilon>0$, by appropriately choosing $\phi$ and $\xi_{i}, i=1, \ldots, N$, for some sufficiently large integer $N$, there exists an $R B F N N W^{* T} \psi(\xi)$ such that

$$
F(\xi)=W^{* T} \psi(\xi)+\Delta(\xi), \quad|\Delta(\xi)| \leq \epsilon, \forall \xi \in \Omega_{\xi},
$$

where $W^{*}$ is the optimal weight vector defined as

$$
W^{*}=\arg \min _{W \in \mathbb{R}^{n}}\left\{\sup _{\xi \in \Omega_{\xi}}\left|\Psi(\xi)-W^{T} \psi(\xi)\right|\right\},
$$

and $\Delta(\xi)$ denotes the approximation error. 


\section{Adaptive Neural Controller Design}

3.1. Performance and Error Transformation Functions. Let the tracking error be defined as

$$
e=y-y_{d}
$$

where $y_{d}$ is the desired trajectory. Similar to $[23,24]$, the mathematical expression of the prescribed tracking performance is given by

$$
-\kappa \varepsilon(t)<e(t)<\tau \varepsilon(t),
$$

where $\kappa$ and $\tau$ are given positive constants and the smooth function is given by

$$
\varepsilon(t)=\left(\varepsilon_{0}-\varepsilon_{\infty}\right) \exp (-l t)+\varepsilon_{\infty},
$$

in which $\varepsilon_{0}$ is the initial value of $\varepsilon(t), \varepsilon_{\infty}$ represents the value of $\varepsilon(t)$ at the steady state, and $l$ is the decreasing rate of $\varepsilon(t)$. Then, introduce the following error transformation:

$$
S(z)=\frac{e(t)}{\varepsilon(t)}
$$

where $z$ is the transformed error and $S(z)$ is a smooth, strictly increasing, and thus invertible function possessing the following properties:

$$
\begin{aligned}
& \lim _{z \rightarrow-\infty} S(z)=-\kappa, \\
& \lim _{z \rightarrow+\infty} S(z)=\tau .
\end{aligned}
$$

Note that if $z$ is kept bounded, we have $-\kappa<S(z)<\tau$, and thus (12) holds. The inverse transformation of $S(z)$ can be written as

$$
z=S^{-1}\left(\frac{e(t)}{\varepsilon(t)}\right):=\Theta\left(\frac{e(t)}{\varepsilon(t)}\right) .
$$

In this paper, we choose

$$
z=\Theta\left(\frac{e(t)}{\varepsilon(t)}\right)=\ln \left(\frac{\kappa+e(t) / \varepsilon(t)}{\tau-e(t) / \varepsilon(t)}\right) .
$$

Differentiating (17) yields

$$
\dot{z}=\eta \dot{y}-\eta v
$$

where $\eta=(\partial \Theta / \partial(e / \varepsilon))(1 / \varepsilon)$ and $v=\dot{y}_{d}+e \dot{\varepsilon} / \varepsilon$. From the properties of the transformation, it is clear that $\eta$ and $v$ are bounded and $0<\eta_{0} \leq \eta$.

Remark 6. From (12) and (13), one can see that $\tau \mathcal{\varepsilon}(0)$ and $-\kappa \varepsilon(0)$ serve as the upper bound of the overshoot and the lower bound of the undershoot of $e(t)$, respectively, the decreasing rate of $\varepsilon(t)$ introduces a lower bound of the convergence rate of $e(t)$, and $\max \left\{\kappa \varepsilon_{\infty}, \tau \varepsilon_{\infty}\right\}$ represents the maximum allowable size of the steady-state value of $e(t)$. Note that $\varepsilon(0), \tau$, and $\kappa$ should be properly chosen such that $-\kappa \varepsilon(0)<e(0)<\tau \varepsilon(0)$.
3.2. Attitude Controller Design via Backstepping. After the error transformation (18), the altitude subsystem (5) is equivalent to

$$
\begin{aligned}
& \dot{z}_{1}=\eta_{1} g_{1}\left(\bar{x}_{1}\right) x_{2}-\eta_{1} v_{1}, \\
& \dot{x}_{2}=f_{2}\left(\bar{x}_{2}\right)+\eta_{2} g_{2}\left(\bar{x}_{2}\right) x_{3}, \\
& \dot{x}_{3}=\eta_{3} g_{3}\left(\bar{x}_{3}\right) x_{4}, \\
& \dot{x}_{4}=\eta_{4} g_{4}\left(\bar{x}_{4}\right) \delta_{E}+f_{4}\left(\bar{x}_{4}\right), \\
& y=x_{1},
\end{aligned}
$$

where $0<\eta_{10} \leq \eta_{1}$ and $\eta_{k}=\eta_{k 0}=1, k=2,3,4$. The stabilization of the transformed system (19) is sufficient to guarantee the prescribed tracking performance of system (5).

Based on the backstepping approach, a trajectory tracking controller is designed for the dynamics model given in (19). The design procedure contains 4 steps, and the actual control law will be deduced at the last step. For convenience, let $F_{i}\left(\xi_{i}\right)$ and $\Omega_{\xi_{i}}$ denote the unknown function to be estimated by RBF NNs and the corresponding compact set in the $i$ th step, respectively. Then by using Lemma 5 , we have

$$
F_{i}\left(\xi_{i}\right)=W_{i}^{* T} \psi_{i}\left(\xi_{i}\right)+\Delta_{i}\left(\xi_{i}\right), \quad\left|\Delta_{i}\left(\xi_{i}\right)\right| \leq \epsilon_{i},
$$

where $\psi_{i}\left(\xi_{i}\right)$ and $\xi_{i}$ denote the vector valued function and the $\mathrm{RBF} N \mathrm{NN}$ input in the step with proper dimensions that are given below.

Step 1. Let $z_{1}$ given by (19) be the first error variable. Define $z_{2}=x_{2}-u_{1}$, where $u_{1}$ is the first virtual control signal. Then the derivative of $z_{1}$ can be expressed as

$$
\dot{z}_{1}=\eta_{1} g_{1}\left(\bar{x}_{1}\right)\left(z_{2}+u_{1}\right)-\frac{1}{2} z_{1}+F_{1}\left(\xi_{1}\right),
$$

where $F_{1}\left(\xi_{1}\right)=(1 / 2) z_{1}-\eta_{1} v_{1}$ and $\xi_{1}:=\left[x_{1}, h_{d}, \dot{h}_{d}, \varepsilon_{1}\right]^{T} \subset$ $\mathbb{R}^{4}$. Since $F_{1}\left(\xi_{1}\right)$ is unknown, we employ an RBF NN to approximate it on a compact set $\Omega_{\xi_{1}} \subset \mathbb{R}^{4}$. By properly choosing the basis function vectors we have

$$
F_{1}\left(\xi_{1}\right)=W_{1}^{* T} \psi_{1}\left(\xi_{1}\right)+\Delta_{1}\left(\xi_{1}\right), \quad\left|\Delta_{1}\left(\xi_{1}\right)\right| \leq \epsilon_{1},
$$

where $\epsilon_{1}$ is a positive constant. With respect to the unknown optimal weight vector in (22), define

$$
\vartheta_{1}=\frac{\left\|W_{1}^{*}\right\|^{2}}{\eta_{10} b_{1}} .
$$

Besides, let $\widehat{\vartheta}_{1}$ be the estimation of $\vartheta_{1}$ and $\widetilde{\vartheta}_{1}:=\widehat{\vartheta}_{1}-\vartheta_{1}$. Consider the first Lyapunov function

$$
L_{1}=\frac{1}{2} z_{1}^{2}+\frac{\eta_{10} b_{1}}{2 \lambda_{1}} \widetilde{\vartheta}_{1}^{2}
$$

Taking the time derivation of (24) yields

$$
\begin{aligned}
\dot{L}_{1}= & z_{1} \eta_{1} g_{1}\left(\bar{x}_{1}\right) z_{2}+z_{1} \eta_{1} g_{1}\left(\bar{x}_{1}\right) u_{1}-\frac{1}{2} z_{1}^{2} \\
& +z_{1} W_{1}^{* T} \psi_{1}\left(\xi_{1}\right)+z_{1} \Delta_{1}\left(\xi_{1}\right)+\frac{\eta_{10} b_{1}}{\lambda_{1}} \widetilde{\vartheta}_{1} \dot{\hat{\vartheta}}_{1} .
\end{aligned}
$$


Using Young's inequality and (23), it can be verified that

$$
\begin{aligned}
z_{1} W_{1}^{* T} \psi_{1}\left(\xi_{1}\right) & \leq \frac{1}{2} z_{1}^{2}\left\|W_{1}^{*}\right\|^{2} \psi_{1}^{T}\left(\xi_{1}\right) \psi_{1}\left(\xi_{1}\right)+\frac{1}{2} \\
& \leq \frac{\eta_{10} b_{1}}{2} \vartheta_{1} z_{1}^{2} \psi_{1}^{T}\left(\xi_{1}\right) \psi_{1}\left(\xi_{1}\right)+\frac{1}{2}, \\
z_{1} \Delta_{1}\left(\xi_{1}\right) & \leq \frac{1}{2} z_{1}^{2}+\frac{1}{2} \epsilon_{1}^{2} .
\end{aligned}
$$

Thus, (25) can be rewritten as

$$
\begin{aligned}
\dot{L}_{1} \leq & z_{1} \eta_{1} g_{1}\left(\bar{x}_{1}\right) z_{2}+z_{1} \eta_{1} g_{1}\left(\bar{x}_{1}\right) u_{1} \\
& +\frac{\eta_{10} b_{1}}{2} \widehat{\vartheta}_{1} z_{1}^{2} \psi_{1}^{T}\left(\xi_{1}\right) \psi_{1}\left(\xi_{1}\right)+\frac{1}{2}+\frac{1}{2} \epsilon_{1}^{2} \\
& +\frac{\eta_{10} b_{1}}{\lambda_{1}} \widetilde{\vartheta}_{1}\left(\dot{\hat{\vartheta}}_{1}-\frac{\lambda_{1}}{2} z_{1}^{2} \psi_{1}^{T}\left(\xi_{1}\right) \psi_{1}\left(\xi_{1}\right)\right),
\end{aligned}
$$

which suggests that we choose the first virtual control signal $u_{1}$ as

$$
u_{1}=-c_{1} z_{1}-\frac{1}{2} \widehat{\vartheta}_{1} z_{1} \psi_{1}^{T}\left(\xi_{1}\right) \psi_{1}\left(\xi_{1}\right)
$$

Let

$$
\dot{\hat{\vartheta}}_{1}=\frac{\lambda_{1}}{2} z_{1}^{2} \psi_{1}^{T}\left(\xi_{1}\right) \psi_{1}\left(\xi_{1}\right)-\lambda_{1} \sigma_{1} \widehat{\vartheta}_{1}, \quad \widehat{\vartheta}_{1}(0) \geq 0
$$

where $c_{1}, \lambda_{1}$, and $\sigma_{1}$ are positive design parameters. Then substituting (28) and (29) into (27), we get

$$
\begin{aligned}
\dot{L}_{1} \leq & -\eta_{10} b_{1} c_{1} z_{1}^{2}+z_{1} \eta_{1} g_{1}\left(\bar{x}_{1}\right) z_{2}-\eta_{10} b_{1} \sigma_{1} \widetilde{\vartheta}_{1} \widehat{\vartheta}_{1}+\frac{1}{2} \\
& +\frac{1}{2} \epsilon_{1}^{2} .
\end{aligned}
$$

Step $i(i=2,3)$. Define $z_{i+1}=x_{i+1}-u_{i}$, where $u_{i}$ is the $i$ th virtual control signal. Then the time derivation of $z_{i}$ is

$$
\begin{aligned}
\dot{z}_{i}= & \eta_{i} g_{i}\left(\bar{x}_{i}\right) z_{i+1}+\eta_{i} g_{i}\left(\bar{x}_{i}\right) u_{i}-\eta_{i-1} g_{i-1}\left(\bar{x}_{i-1}\right) z_{i-1} \\
& -\frac{1}{2} z_{i}+F_{i}\left(\xi_{i}\right),
\end{aligned}
$$

where $F_{i}\left(\xi_{i}\right)=f_{i}\left(\bar{x}_{i}\right)+\eta_{i-1} g_{i-1}\left(\bar{x}_{i-1}\right) z_{i-1}+(1 / 2) z_{i}-\dot{u}_{i-1}$ and $\xi_{i}=\left[\bar{x}_{i}, h_{d}, \ldots, h_{d}^{(i)}, \widehat{\vartheta}_{1}, \ldots, \widehat{\vartheta}_{i}, \varepsilon_{1}\right]^{T} \in \Omega_{\xi_{i}}$. Consider the $i$ th Lyapunov function

$$
L_{i}=L_{i-1}+\frac{1}{2} z_{i}^{2}+\frac{\eta_{i 0} b_{i}}{2 \lambda_{i}} \widetilde{\vartheta}_{i}^{2}
$$

where $\lambda_{i}$ is a positive design parameter, $\widetilde{\vartheta}_{i}=\widehat{\vartheta}_{i}-\vartheta_{i}$ with $\vartheta_{i}=$ $\left\|W_{i}^{*}\right\|^{2} / \eta_{i 0} b_{i}$. By taking the time derivation of (32), we have

$$
\begin{aligned}
\dot{L}_{i}= & \dot{L}_{i-1}-\eta_{i-1} g_{i-1}\left(\bar{x}_{i-1}\right) z_{i-1} z_{i}+z_{i} \eta_{i} g_{i}\left(\bar{x}_{i}\right) z_{i} \\
& +z_{i} \eta_{i} g_{i}\left(\bar{x}_{i}\right) u_{i}-\frac{1}{2} z_{i}^{2}+z_{i} F_{i}\left(\xi_{i}\right)+\frac{\eta_{i 0} b_{i}}{\lambda_{i}} \widetilde{\vartheta}_{i} \dot{\widehat{\vartheta}}_{i} .
\end{aligned}
$$

Similar to (26), we have

$$
\begin{aligned}
\dot{L}_{i} \leq & \dot{L}_{i-1}-\eta_{i-1} g_{i-1}\left(\bar{x}_{i-1}\right) z_{i-1} z_{i}+z_{i} \eta_{i} g_{i}\left(\bar{x}_{i}\right) z_{i+1} \\
& +z_{i} \eta_{i} g_{i}\left(\bar{x}_{i}\right) u_{i}+\frac{\eta_{i 0} b_{i}}{2} \widehat{\vartheta}_{i} z_{i}^{2} \psi_{i}^{T}\left(\xi_{i}\right) \psi_{i}\left(\xi_{i}\right) \\
& +\frac{\eta_{i 0} b_{i}}{\lambda_{i}} \widetilde{\vartheta}_{i}\left(\dot{\widehat{\vartheta}}_{i}-\frac{\lambda_{i}}{2} z_{i}^{2} \psi_{i}^{T}\left(\xi_{i}\right) \psi_{i}\left(\xi_{i}\right)\right)+\frac{1}{2}+\frac{1}{2} \epsilon_{i}^{2} .
\end{aligned}
$$

Choose the $i$ th virtual control signal

$$
u_{i}=-c_{i} z_{i}-\frac{1}{2} \widehat{\vartheta}_{i} z_{i} \psi_{i}^{T}\left(\xi_{i}\right) \psi_{i}\left(\xi_{i}\right)
$$

where $\widehat{\vartheta}_{i}$ is updated by

$$
\dot{\hat{\vartheta}}_{i}=\frac{\lambda_{i}}{2} z_{i}^{2} \psi_{i}^{T}\left(\xi_{i}\right) \psi_{i}\left(\xi_{i}\right)-\lambda_{i} \sigma_{i} \widehat{\vartheta}_{i}, \quad \widehat{\vartheta}_{i}(0) \geq 0,
$$

with $c_{i}, \lambda_{i}$, and $\sigma_{i}$ being positive design parameters. Substituting (35), (36), and (30) into (34), we get

$$
\begin{aligned}
\dot{L}_{i} \leq & -\sum_{k=1}^{i} \eta_{k 0} b_{k} c_{k} z_{k}^{2}+z_{i} \eta_{i} g_{i}\left(\bar{x}_{i}\right) z_{i+1}-\sum_{k=1}^{i} \eta_{k 0} b_{k} \sigma_{k} \widetilde{\vartheta}_{k} \widehat{\vartheta}_{k} \\
& +\sum_{k=1}^{i} \frac{1}{2}\left(1+\epsilon_{k}^{2}\right) .
\end{aligned}
$$

Step 4 . The time derivative of $z_{4}$ is

$$
\dot{z}_{4}=-\eta_{3} g_{3}\left(\bar{x}_{3}\right) z_{3}+\eta_{4} g_{4}\left(\bar{x}_{4}\right) \delta_{E}-\frac{1}{2} z_{4}+F_{4}\left(\xi_{4}\right),
$$

where $F_{4}\left(\xi_{4}\right)=f_{4}\left(\bar{x}_{4}\right)+\eta_{3} g_{3}\left(\bar{x}_{3}\right) z_{3}+(1 / 2) z_{4}-\dot{u}_{3}$ and $\xi_{4}=$ $\left[\bar{x}_{4}, h_{d}, \ldots, h_{d}^{(4)}, \widehat{\vartheta}_{1}, \widehat{\vartheta}_{2}, \widehat{\vartheta}_{3}, \varepsilon_{h}\right]^{T} \in \Omega_{\xi_{4}} \subset \mathbb{R}^{13}$. Let

$$
L_{4}=L_{3}+\frac{1}{2} z_{4}^{2}+\frac{\eta_{40} b_{4}}{2 \lambda_{4}} \widetilde{\vartheta}_{4}^{2}
$$

where $\lambda_{4}$ is a positive design parameter and $\widetilde{\vartheta}_{4}=\widehat{\vartheta}_{4}-\vartheta_{4}$ with $\vartheta_{4}=\left\|W_{4}^{*}\right\|^{2} / \eta_{40} b_{4}$. Differentiating (39) we have

$$
\begin{aligned}
\dot{L}_{4}= & \dot{L}_{3}+\frac{\eta_{40} b_{4}}{\lambda_{4}} \widetilde{\vartheta}_{4} \dot{\hat{\vartheta}}_{4}-z_{4} \eta_{3} g_{3}\left(\bar{x}_{3}\right) z_{3} \\
& +z_{4} \eta_{4} g_{4}\left(\bar{x}_{4}\right) \delta_{E}-\frac{1}{2} z_{4}^{2}+z_{4} F_{4}\left(\xi_{4}\right) .
\end{aligned}
$$

Similar to (26), (40) can be rewritten as

$$
\begin{aligned}
\dot{L}_{4} \leq & \dot{L}_{3}-z_{4} \eta_{3} g_{3}\left(\bar{x}_{3}\right) z_{3}+z_{4} \eta_{4} g_{4}\left(\bar{x}_{4}\right) \delta_{E} \\
& +\frac{\eta_{40} b_{4}}{2} \widehat{\vartheta}_{4} z_{4}^{2} \psi_{4}^{T}\left(\xi_{4}\right) \psi_{4}\left(\xi_{4}\right)+\frac{1}{2}+\frac{1}{2} \epsilon_{4}^{2} \\
& +\frac{\eta_{40} b_{4}}{\lambda_{4}} \widetilde{\vartheta}_{4}\left(\dot{\widehat{\vartheta}}_{4}-\frac{\lambda_{4}}{2} z_{4}^{2} \psi_{4}^{T}\left(\xi_{4}\right) \psi_{4}\left(\xi_{4}\right)\right) .
\end{aligned}
$$

Choose the control signal

$$
\delta_{E}=-c_{4} z_{4}-\frac{1}{2} \widehat{\vartheta}_{4} z_{4} \psi_{4}^{T}\left(\xi_{4}\right) \psi_{4}\left(\xi_{4}\right),
$$


where $\widehat{\vartheta}_{4}$ is updated by

$$
\dot{\widehat{\vartheta}}_{4}=\frac{\lambda_{4}}{2} z_{4}^{2} \psi_{4}^{T}\left(\xi_{4}\right) \psi_{4}\left(\xi_{4}\right)-\lambda_{4} \sigma_{4} \widehat{\vartheta}_{4}, \quad \widehat{\vartheta}_{4}(0) \geq 0
$$

with $c_{4}, \lambda_{4}$, and $\sigma_{4}$ being positive design parameters. Substituting (42), (43), and (37) into (41), we arrive at

$$
\begin{aligned}
\dot{L}_{4} \leq & -\sum_{k=1}^{4} c_{k} \eta_{k 0} b_{k} z_{k}^{2}-\sum_{k=1}^{4} \eta_{k 0} b_{k} \sigma_{k} \widetilde{\vartheta}_{k} \widehat{\vartheta}_{k} \\
& +\sum_{k=1}^{4}\left(\frac{1}{2}+\frac{1}{2} \epsilon_{k}^{2}\right) .
\end{aligned}
$$

Remark 7. The RBF NNs are used to compensate for the complex and uncertain terms to solve the problem of controller complexity, and the repeated derivation of virtual control signal $u_{i}$ can be avoided. Compared with the neural based control $[16,21]$, in each design step, by using the estimation of the norm of the NNs weights, only one parameter needs to be updated online; therefore the design procedure can be greatly simplified and the computational burden is greatly reduced. Moreover, the lower bound of the virtual control coefficient $\eta_{i} g_{i}$ is used to avoid the singularity problem without any additional effort.

Remark 8. Since the approximation ability of RBF NNs is on a compact set, we can only guarantee the semiglobal stability of the control scheme.

Theorem 9. Consider system (5) under the Assumptions 2 and 4 , with the error transformation (17), the virtual control signals (28) and (35), the control law (42), and the adaptive laws (29), (36), and (43). Then all closed-loop signals are uniformly bounded and the prescribed tracking performance (12) can be guaranteed.

Proof. Using the following facts:

$$
-\widetilde{\vartheta}_{i} \widehat{\vartheta}_{i} \leq-\frac{1}{2} \widetilde{\vartheta}_{i}^{2}+\frac{1}{2} \widehat{\vartheta}_{i}^{2}, \quad i=1, \ldots, 4,
$$

we rewrite (44) as

$$
\dot{L}_{4} \leq-\sum_{k=1}^{4} c_{k} \eta_{k 0} b_{k} z_{k}^{2}-\sum_{k=1}^{4} \frac{1}{2} \eta_{k 0} b_{k} \sigma_{k} \widetilde{\vartheta}_{k}^{2}+\varsigma,
$$

where

$$
\varsigma=\sum_{k=1}^{4} \frac{1}{2} \eta_{k 0} b_{k} \sigma_{k} \widehat{\vartheta}_{k}^{2}+\sum_{k=1}^{4}\left(\frac{1}{2}+\frac{1}{2} \epsilon_{k}^{2}\right) .
$$

Let

$$
\hbar=\min \left\{2 \eta_{i 0} b_{i} c_{i}, \lambda_{i} \sigma_{i}, i=1, \ldots, 4\right\} .
$$

Then we have

$$
\dot{L}_{4} \leq-\hbar L_{4}+\varsigma
$$

Solving (49) gives

$$
0 \leq L_{4}(t) \leq \frac{\varsigma}{\hbar}+\left(L_{4}(0)-\frac{\varsigma}{\hbar}\right) e^{-\hbar t}, \quad t \geq 0
$$

which implies that $L_{4}, z_{i}, \widetilde{\vartheta}_{i}$, and $\widehat{\vartheta}_{i}$ are bounded. Since $z_{1}$ is bounded, according to the error transformation of (15), to (17) we can obtain that $-\kappa<S(z)<\tau$; as a result, we have $-\kappa \varepsilon(t)<$ $e(t)<\tau \varepsilon(t)$; that is, the prescribed tracking performance is guaranteed. This completes the proof.

3.3. Velocity Controller Design via Dynamic Inversion. The velocity subsystem of (1) can be rewritten as follows:

$$
\dot{V}=f_{V}\left(\bar{x}_{4}, V\right)+g_{V}\left(\bar{x}_{4}, V\right) \beta_{c},
$$

where $f_{V}\left(\bar{x}_{4}, V\right)$ and $g_{V}\left(\bar{x}_{4}, V\right)$ are unknown nonlinear function $g_{V}\left(\bar{x}_{4}, V\right) \geq b_{V}>0$. Then define the velocity tracking error as $e_{V}=V-V_{d}$. According to (17) and (18) we obtain

$$
\dot{z}_{V}=-\eta_{V} v_{V}+\eta_{V} f_{V}\left(\bar{x}_{4}, V\right)+\eta_{V} g_{V}\left(\bar{x}_{4}, V\right) \beta_{c} .
$$

We assume that $0<\eta_{V 0} \leq \eta_{V}$. The transformed system dynamics of (52) can be rewritten as

$$
\dot{z}_{V}=\eta_{V} g_{V}\left(\bar{x}_{4}, V\right) \beta_{c}-\frac{1}{2} z_{V}+F_{V}\left(\xi_{V}\right),
$$

where $F_{V}\left(\xi_{V}\right)=(1 / 2) z_{V}-\eta_{V} v_{V}+\eta_{V} f_{V}\left(\bar{x}_{4}, V\right)$ and $\xi_{V}:=$ $\left[\bar{x}_{4}, V, \dot{V}_{d}, \varepsilon_{V}\right]^{T} \in \Omega_{\xi_{V}}$. Since $F_{V}\left(\xi_{V}\right)$ is an unknown nonlinear function, we use an RBF NN to approximate it:

$$
F_{V}\left(\xi_{V}\right)=W_{V}^{* T} \psi_{V}\left(\xi_{V}\right)+\Delta_{V}\left(\xi_{V}\right), \quad\left|\Delta_{V}\left(\xi_{V}\right)\right| \leq \epsilon_{V} .
$$

The control law and the adaptive update law are designed as follows:

$$
\begin{gathered}
\beta_{c}=-c_{V} z_{V}-\frac{1}{2} z_{V} \widehat{\vartheta}_{V} \psi_{V}^{T}\left(\xi_{V}\right) \psi_{V}\left(\xi_{V}\right), \\
\dot{\widehat{\vartheta}}_{V}=\frac{\lambda_{V}}{2} z_{V}^{2} \psi_{V}^{T}\left(\xi_{V}\right) \psi_{V}\left(\xi_{V}\right)-\lambda_{V} \sigma_{V} \widehat{\vartheta}_{V},
\end{gathered}
$$

where $\widehat{\vartheta}_{V}$ is the estimate of $\vartheta_{V}=\left\|W_{V}^{*}\right\|^{2} / \eta_{V 0} b_{V}$, and $c_{V}$, $\gamma_{V}$, and $\sigma_{V}$ are positive design parameters. Consider the Lyapunov function

$$
L_{V}=\frac{1}{2} z_{V}^{2}+\frac{\eta_{V 0} b_{V}}{2 \lambda_{V}} \widetilde{\vartheta}_{V}^{2}
$$

Differentiating $L_{V}$ we have

$$
\begin{aligned}
\dot{L}_{V} \leq & z_{V} \eta_{V 0} b_{V} \beta_{c}+\frac{\eta_{V 0} b_{V}}{2} z_{V}^{2} \widehat{\vartheta} \psi_{V}^{T}\left(\xi_{1}\right) \psi_{V}\left(\xi_{V}\right) \\
& +\frac{\eta_{V 0} b_{V}}{\lambda_{V}} \widetilde{\vartheta}_{V}\left(\dot{\hat{\vartheta}}_{V}-\frac{\lambda_{V}}{2} z_{V}^{2} \psi_{V}^{T}\left(\xi_{1}\right) \psi_{V}\left(\xi_{V}\right)\right)+\frac{1}{2} \\
& +\frac{1}{2} \epsilon_{V}^{2} .
\end{aligned}
$$

Substituting (55) into (57) then

$$
\dot{L}_{V} \leq-c_{V} \eta_{V 0} b_{V} z_{V}^{2}-\eta_{V 0} b_{V} \sigma_{V} \widetilde{\vartheta}_{V} \widehat{\vartheta}_{V}+\frac{1}{2}+\frac{1}{2} \epsilon_{V}^{2},
$$


with inequation

$$
-\widetilde{\vartheta}_{V} \widehat{\vartheta}_{V} \leq-\frac{1}{2} \widetilde{\vartheta}_{V}^{2}+\frac{1}{2} \widehat{\vartheta}^{2}
$$

and then

$$
\begin{aligned}
\dot{L}_{V} \leq & -c_{V} \eta_{V 0} b_{V} z_{V}^{2}-\frac{1}{2} \eta_{V 0} b_{V} \sigma_{V} \widetilde{\vartheta}_{V}^{2}+\frac{1}{2} \eta_{V 0} b_{V} \sigma_{V} \widehat{\vartheta}^{2} \\
& +\frac{1}{2}+\frac{1}{2} \epsilon_{V}^{2} \\
\leq & -\kappa_{V} L_{V}+\mu_{V},
\end{aligned}
$$

where

$$
\begin{aligned}
& \kappa_{V}=\min \left(2 c_{V} \eta_{V 0} b_{V}, \lambda_{V} \sigma_{V}\right), \\
& \mu_{V}=\frac{1}{2} \eta_{V 0} b_{V} \sigma_{V} \widehat{\vartheta}^{2}+\frac{1}{2}+\frac{1}{2} \epsilon_{V}^{2} .
\end{aligned}
$$

Solving (60) gives

$$
0 \leq L_{V}(t) \leq \frac{\mu_{V}}{\kappa_{V}}+\left(L_{V}(0)-\frac{\mu_{V}}{\kappa_{V}}\right) e^{-\kappa_{V} t}, \quad t \geq 0 .
$$

It is clear that $z_{V}, \widehat{\vartheta}_{V}$, and $\widetilde{\vartheta}_{V}$ are bounded. Owning that $z_{V}$ is bounded, together with the error transformation of (11) into (17), implies that the prescribed tracking performance is guaranteed.

\section{Simulation Results}

In this section, the numerical simulation results are presented to show the performance of the control scheme. Simulation of the HFV model is conducted for trimmed cruise conditions of $110000 \mathrm{ft}$ and Mach 15. The parameters of simulation model are taken from $[16,21]$. The control objective is to track the step change of $100 \mathrm{ft} / \mathrm{s}$ in airspeed and $2000 \mathrm{ft}$ in altitude. Linear command filters are used to generate the differentiable commands:

$$
\begin{aligned}
\frac{h_{d}}{h_{c}} & =\frac{\omega_{n_{1}}^{2} \omega_{n_{2}}^{2}}{\left(p+\Phi_{n_{1}}\right)^{2}\left(p^{2}+2 \zeta \Phi_{n_{2}} p+\Phi_{n_{2}}^{2}\right)}, \\
\frac{V_{d}}{V_{c}} & =\frac{\omega_{n_{1}}^{2}}{p^{2}+2 \zeta \omega_{n_{1}} p+\omega_{n_{1}}^{2}},
\end{aligned}
$$

where $p$ is Laplace operator, $\oplus_{n 1}=0.5, \Phi_{n 2}=0.3$, and $\zeta_{h}=0.95 . h_{c}$ and $V_{c}$ are step commands. The inputs of the RBF NNs are $\xi_{1}, \xi_{2}, \xi_{3}, \xi_{4}$, and $\xi_{V}$ and the nonlinear functions are approximated with width $\phi=1$. The initial values of the adaptive laws are $\widehat{\vartheta}_{1}(0)=\widehat{\vartheta}_{2}(0)=\widehat{\vartheta}_{3}(0)=\widehat{\vartheta}_{4}(0)=\widehat{\vartheta}_{V}(0)=$ 0 . In addition we choose the design parameters $c_{1}=0.04$, $c_{2}=150, c_{3}=120, c_{4}=100, c_{V}=0.5, \lambda_{1}=0.1, \lambda_{2}=0.1$, $\lambda_{3}=0.01, \lambda_{4}=0.01, \lambda_{V}=10, \sigma_{1}=0.1, \sigma_{2}=0.1, \sigma_{3}=0.1$, $\sigma_{4}=0.1$, and $\sigma_{V}=1$. The parameters of performance functions are given by $\varepsilon_{h 0}=30, \varepsilon_{h \infty}=5, l_{h}=0.2, \varepsilon_{V 0}=20$, $\varepsilon_{V \infty}=2, l_{V}=0.5$, and $\kappa=\tau=1$. The initial states are chosen as $V=15060 \mathrm{ft} / \mathrm{s}, h=110000 \mathrm{ft}, \gamma=0 \mathrm{rad}, \theta=0.0315 \mathrm{rad}$, and $q=0 \mathrm{rad} / \mathrm{s}$.

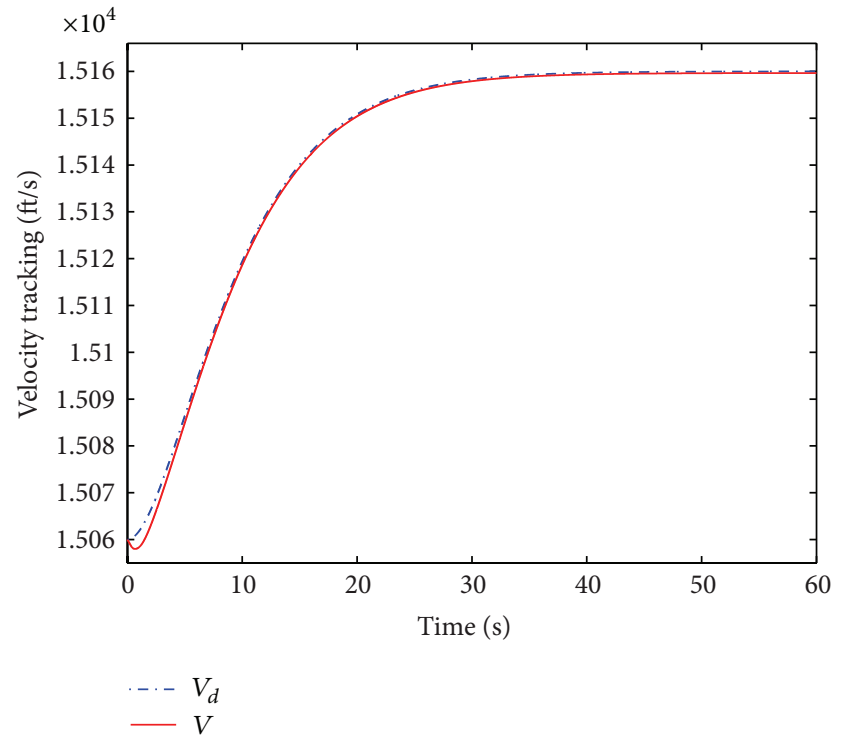

FIGURE 1: Velocity tracking.

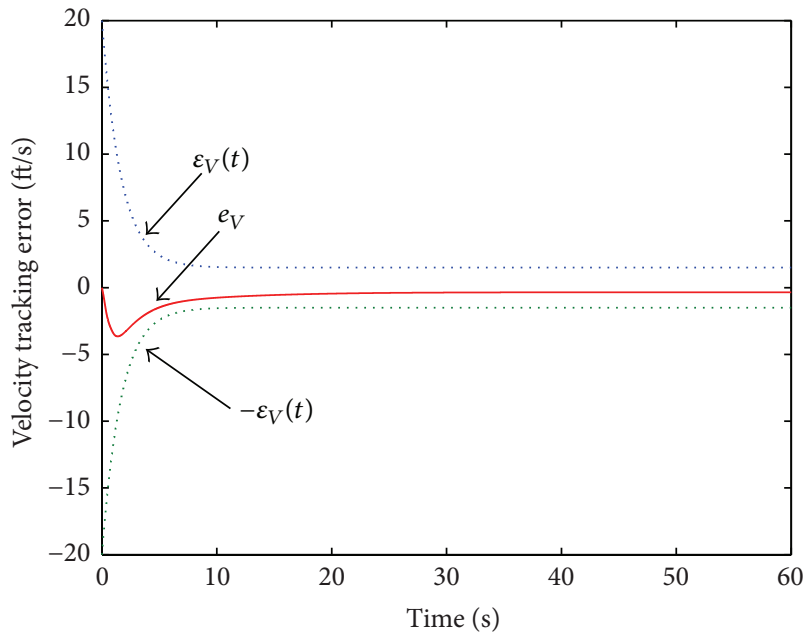

FIGURE 2: The velocity tracking error $e_{V}$ and the prescribed error bounds.

The simulation results are presented in Figures 1-10. The responses to $100-\mathrm{ft} / \mathrm{s}$ step-velocity and $2000-\mathrm{ft}$ step-aliunde command in trimmed condition are depicted in Figures 1-3 and Figures 4-6, respectively. From Figures 2 and 5, we see that the tracking errors performance are guaranteed. Figures 7-10 show the simulation results of altitude tracking with square wave trajectory. From the results of simulations, the maximum value of terms $T \sin \alpha /(\mathrm{mV})$ is $3 \times 10^{-5}$; it is much smaller than the term $L /(m V)$ whose minimum value is $1 \times$ $10^{-3}$ and the maximum value of flight path angle $\gamma$ is less than $0.012 \mathrm{rad}$. Therefore, Assumption 1 is reasonable.

\section{Conclusion}

An adaptive neural control scheme has been proposed for a class of longitudinal dynamics of a generic hypersonic 


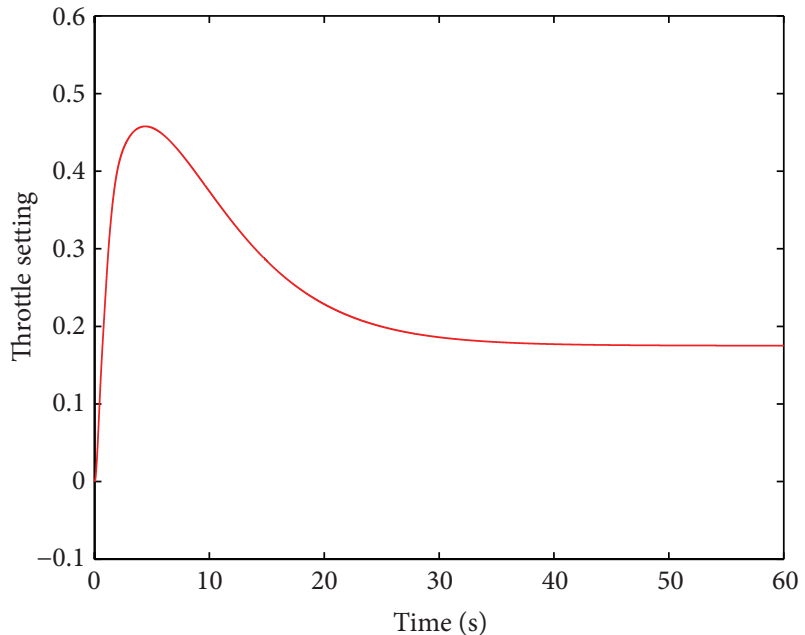

FIgURE 3: Throttle setting $\beta$.

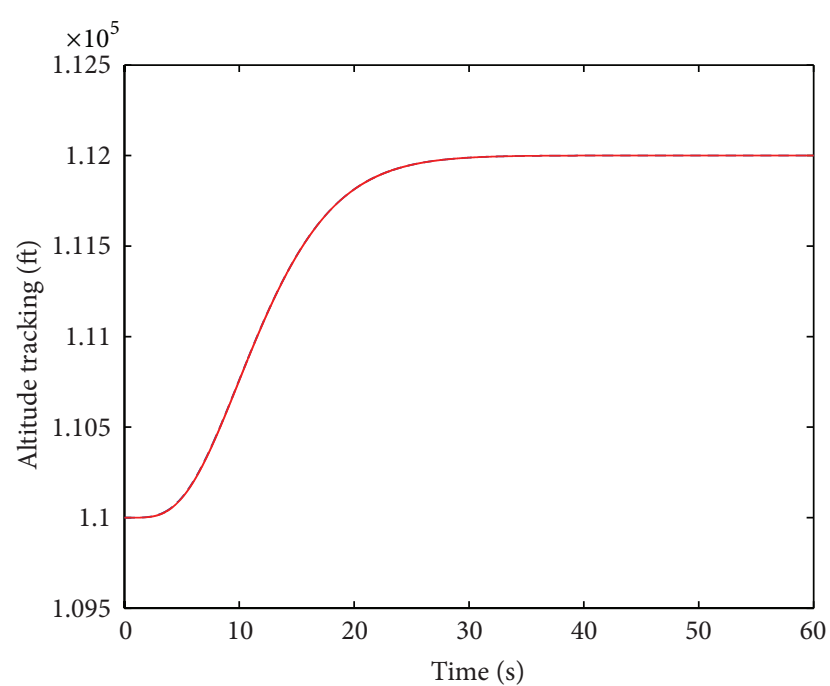

$--h_{d}$

FIgUre 4: The altitude tracking.

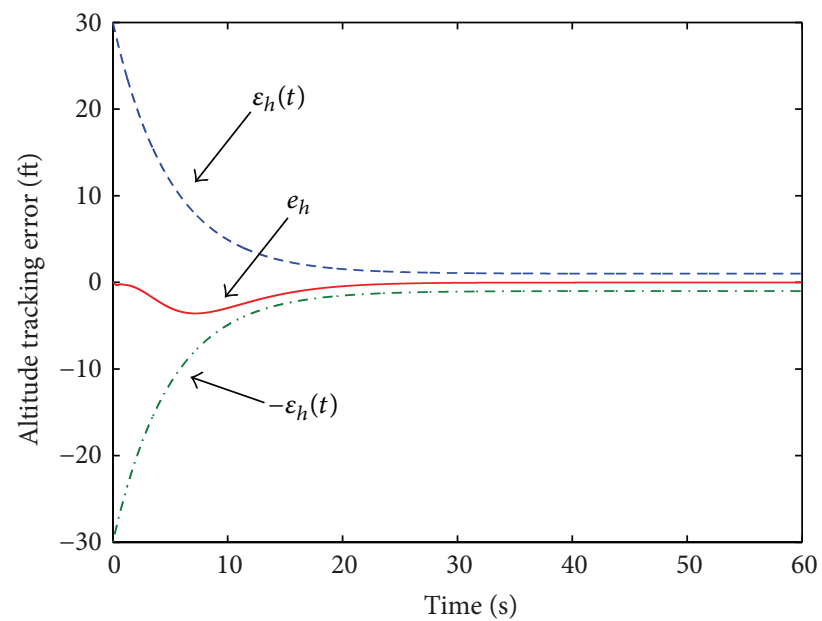

Figure 5: Altitude tracking error $e_{h}$ and the prescribed error bounds.

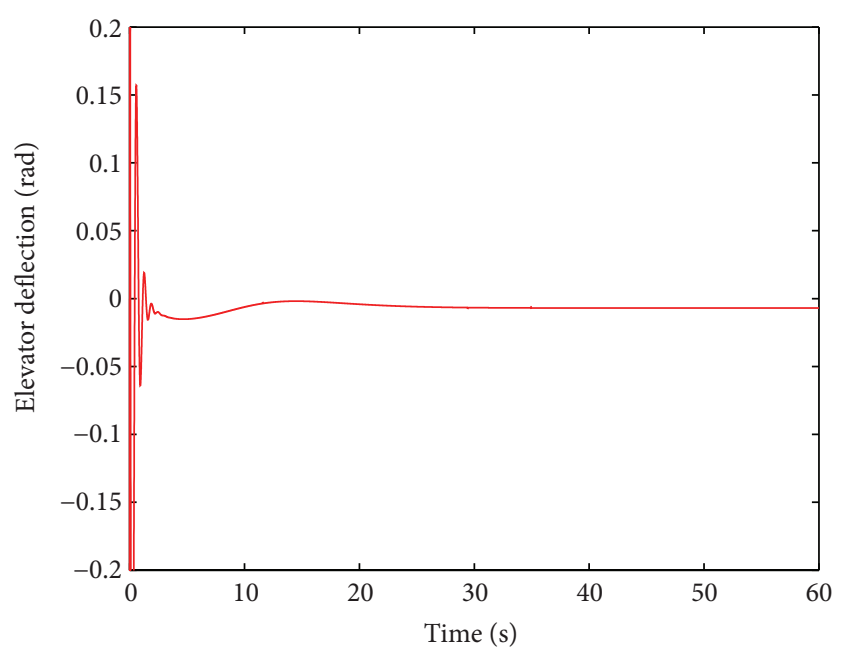

Figure 6: Elevator deflection $\delta_{E}$.

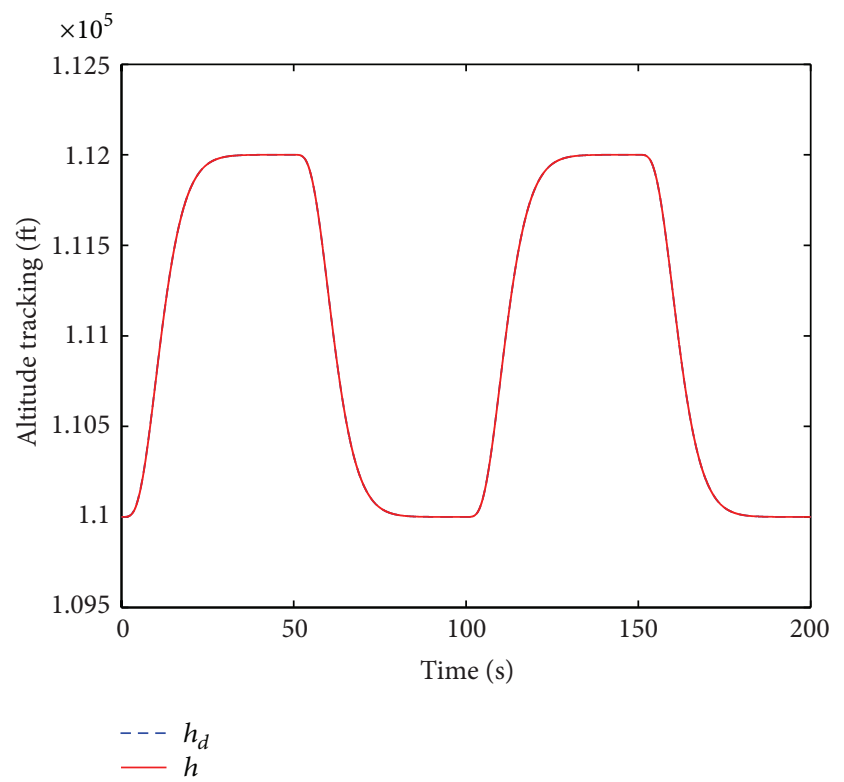

FIgURE 7: Altitude tracking.

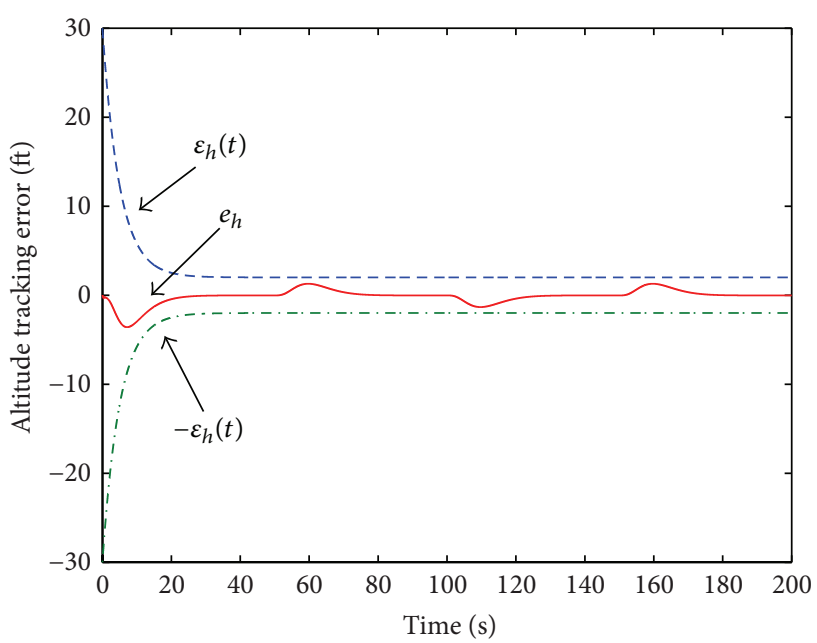

FIGURE 8: Altitude tracking error $e_{h}$ and the prescribed error bounds. 


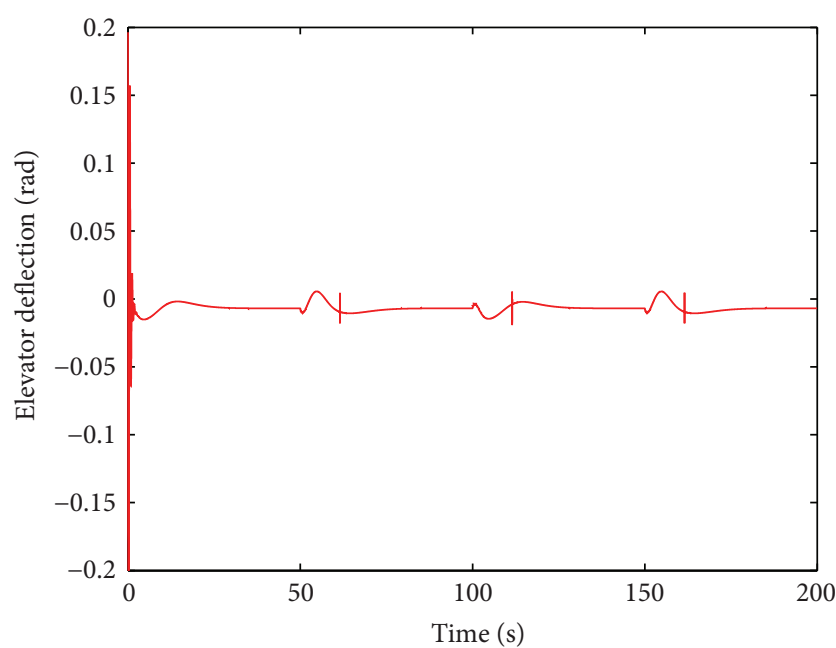

Figure 9: Elevator deflection $\delta_{E}$.

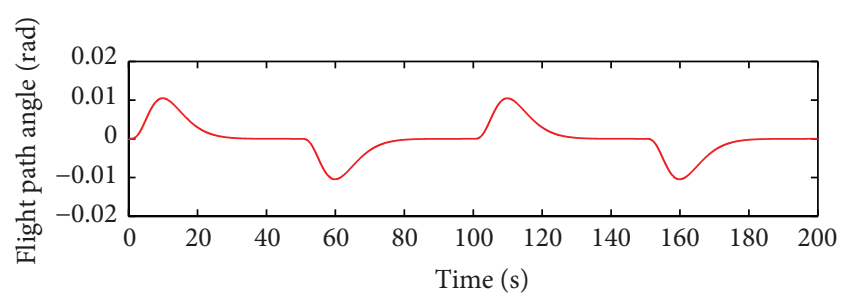

(a)

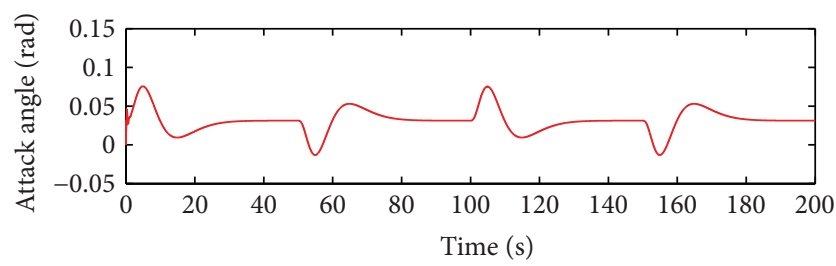

(b)

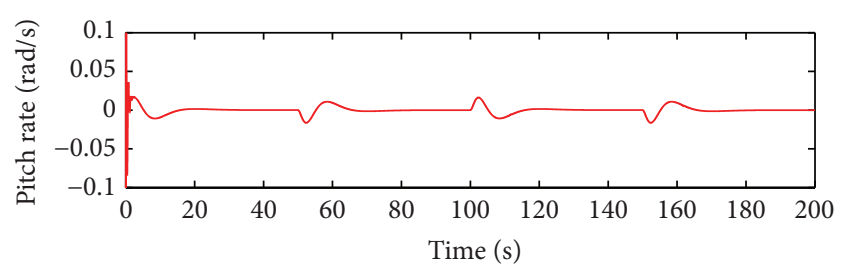

(c)

Figure 10: (a) Path angel $\gamma$; (b) attack angel $\alpha$; (c) pitch rate $q$.

flight vehicle. We have shown that, by using a new constraint variable, the prescribed tracking performance can be achieved. The unknown nonlinear functions associated with each recursive step of backstepping control were approximated by using RBF NNs. For each design step, only one parameter needs to be updated online. Thus the explosion of the complex problem in backstepping control scheme and the computational burden can be greatly reduced. Numerical simulations revealed that the tracking error clearly satisfies the prescribed performance specification and verified the proposed design scheme. Currently, we assume that all of the system states are available and the controller is based on state feedback. However, some states cannot be obtained in some circumstances, especially when the sensor fault occurs. As a result, future work will be focused on output feedback control law design.

\section{Conflict of Interests}

The authors declare that there is no conflict of interests regarding the publication of this paper.

\section{Acknowledgments}

This work was supported by the National Natural Science Foundation of China (Grant no. 61374048), the Research Fund for Doctoral Program of Higher Education of China (Grant no. 20121102110008), and the China Postdoctoral Science Foundation (Grant no. 2013M540839).

\section{References}

[1] C. I. Marrison and R. F. Stengel, "Design of robust control systems for a hypersonic aircraft," Journal of Guidance, Control, and Dynamics, vol. 21, no. 1, pp. 58-63, 1998.

[2] H. Xu, M. Mirmirani, and P. A. Ioannou, "Robust neural adaptive control of a hypersonic aircraft," in Proceedings of the AIAA Guidance, Navigation, and Control Conference, vol. 7, pp. 1-11, AIAA, Austin, Tex, USA, August 2003.

[3] H. Xu, M. D. Mirmirani, and P. A. Ioannou, "Adaptive sliding mode control design for a hypersonic flight vehicle," Journal of Guidance, Control, and Dynamics, vol. 27, no. 5, pp. 829-838, 2004.

[4] H. Li, P. Lin, and D. Xu, "Control-oriented modeling for airbreathing hypersonic vehicle using parameterized configuration approach," Chinese Journal of Aeronautics, vol. 24, no. 1, pp. 81-89, 2011

[5] X. Hu, L. Wu, C. Hu, and H. Gao, "Adaptive sliding mode tracking control for a flexible air-breathing hypersonic vehicle," Journal of the Franklin Institute. Engineering and Applied Mathematics, vol. 349, no. 2, pp. 559-577, 2012.

[6] D. Gao and Z. Sun, "Fuzzy tracking control design for hypersonic vehicles via ts model," Science China. Information Sciences, vol. 54, no. 3, pp. 521-528, 2011.

[7] D. Gao, S. Wang, and D. Lu, "Adaptive neural control design for hypersonic aircraft using time scale separation," in Proceedings of the International Conference on Unmanned Aircraft Systems (ICUAS '13), pp. 1037-1042, May 2013.

[8] B. Xu, D. Gao, and S. Wang, "Adaptive neural control based on HGO for hypersonic flight vehicles," Science China Information Sciences, vol. 54, no. 3, pp. 511-520, 2011.

[9] X. Hu, L. Wu, C. Hu, Z. Wang, and H. Gao, "Dynamic output feedback control of a flexible air-breathing hypersonic vehicle via T-S fuzzy approach," International Journal of Systems Science, vol. 45, no. 8, pp. 1740-1756, 2014.

[10] D. O. Sigthorsson and A. Serrani, "Development of linear parameter-varying models of hypersonic air-breathing vehicles," in Proceedings of the AIAA Guidance, Navigation, and Control Conference, August 2009. 
[11] J. T. Parker, A. Serrani, S. Yurkovich, M. A. Bolender, and D. B. Doman, "Control-oriented modeling of an air-breathing hypersonic vehicle," Journal of Guidance, Control, and Dynamics, vol. 30, no. 3, pp. 856-869, 2007.

[12] O. U. Rehman, B. Fidan, and I. R. Petersen, "Uncertainty modeling and robust minimax LQR control of multivariable nonlinear systems with application to hypersonic flight," Asian Journal of Control, vol. 14, no. 5, pp. 1180-1193, 2012.

[13] O. Ur Rehman, I. R. Petersen, and B. Fidan, "Feedback linearization-based robust nonlinear control design for hypersonic flight vehicles," Proceedings of the Institution of Mechanical Engineers. Part I: Journal of Systems and Control Engineering, vol. 227, no. 1, pp. 3-11, 2013.

[14] Q. Wang and R. F. Stengel, "Robust nonlinear control of a hypersonic aircraft," Journal of Guidance, Control, and Dynamics, vol. 23, no. 4, pp. 577-585, 2000.

[15] L. Fiorentini, A. Serrani, M. A. Bolender, and D. B. Doman, "Nonlinear robust adaptive control of flexible air-breathing hypersonic vehicles," Journal of Guidance, Control, and Dynamics, vol. 32, no. 2, pp. 401-416, 2009.

[16] B. Xu, F. Sun, C. Yang, D. Gao, and J. Ren, "Adaptive discretetime controller design with neural network for hypersonic flight vehicle via back-stepping," International Journal of Control, vol. 84, no. 9, pp. 1543-1552, 2011.

[17] R. M. Sanner and J. J. E. Slotine, "Gaussian networks for direct adaptive control," IEEE Transactions on Neural Networks, vol. 3, no. 6, pp. 837-863, 1992.

[18] D. Gao, Z. Sun, and T. Du, "Dynamic surface control for hypersonic aircraft using fuzzy logic system," in Proceedings of the IEEE International Conference on Automation and Logistics (ICAL '07), pp. 2314-2319, IEEE, August 2007.

[19] B. Xu, D. Wang, F. Sun, and Z. Shi, "Direct neural control of hypersonic flight vehicles with prediction model in discrete time," Neurocomputing, vol. 115, pp. 39-48, 2013.

[20] W. A. Butt, L. Yan, and A. S. Kendrick, "Adaptive dynamic surface control of a hypersonic flight vehicle with improved tracking," Asian Journal of Control, vol. 15, no. 2, pp. 594-605, 2013.

[21] W. A. Butt, L. Yan, and A. S. Kendrick, "Dynamic surface control for nonlinear hypersonic air vehicle using neural network," in Proceedings of the 29th Chinese Control Conference (CCC '10), pp. 733-738, July 2010.

[22] T. Lee and Y. Kim, "Nonlinear adaptive flight control using backstepping and neural networks controller," Journal of Guidance, Control, and Dynamics, vol. 24, no. 4, pp. 675-682, 2001.

[23] C. P. Bechlioulis and G. A. Rovithakis, "Prescribed performance adaptive control for multi-input multi-output affine in the control nonlinear systems," IEEE Transactions on Automatic Control, vol. 55, no. 5, pp. 1220-1226, 2010.

[24] C. P. Bechlioulis and G. A. Rovithakis, "Adaptive control with guaranteed transient and steady state tracking error bounds for strict feedback systems," Automatica, vol. 45, no. 2, pp. 532-538, 2009.

[25] S. Tong, S. Sui, and Y. Li, "Fuzzy adaptive output feedback control of MIMO nonlinear systems with partial tracking errors constrained," IEEE Transactions on Fuzzy Systems, 2014.

[26] J. Wu, W. Chen, and J. Li, "Fuzzy-approximation-based global adaptive control for uncertain strict-feedback systems with a priori known tracking accuracy," Fuzzy Sets and Systems, vol. 273, pp. 1-25, 2015.
[27] J. Wu, W. Chen, F. Yang, J. Li, and Q. Zhu, "Global adaptive neural control for strict-feedback time-delay systems with predefined output accuracy," Information Sciences, vol. 301, pp. 27-43, 2015.

[28] J. Wu, J. Li, and W. Chen, "Semi-globally/globally stable adaptive nn backstepping control for uncertain mimo systems with tracking accuracy known a priori," Journal of the Franklin Institute, vol. 351, no. 12, pp. 5274-5309, 2014.

[29] S.-C. Tong, Y.-M. Li, G. Feng, and T.-S. Li, “Observer-based adaptive fuzzy backstepping dynamic surface control for a class of MIMO nonlinear systems," IEEE Transactions on Systems, Man, and Cybernetics, Part B: Cybernetics, vol. 41, no. 4, pp. 1124-1135, 2011.

[30] Y. Li, S. Tong, and T. Li, “Observer-based adaptive fuzzy tracking control of mimo stochastic nonlinear systems with unknown control direction and unknown dead-zones," IEEE Transactions on Fuzzy Systems, 2014.

[31] C. Wang and Y. Lin, "Multivariable adaptive backstepping control: a norm estimation approach," IEEE Transactions on Automatic Control, vol. 57, no. 4, pp. 989-995, 2012.

[32] B. Chen, X. Liu, K. Liu, and C. Lin, "Direct adaptive fuzzy control of nonlinear strict-feedback systems," Automatica, vol. 45, no. 6, pp. 1530-1535, 2009.

[33] Q. Zhao and Y. Lin, "Adaptive fuzzy dynamic surface control with prespecified tracking performance for a class of nonlinear systems," Asian Journal of Control, vol. 13, no. 6, pp. 1082-1091, 2011. 


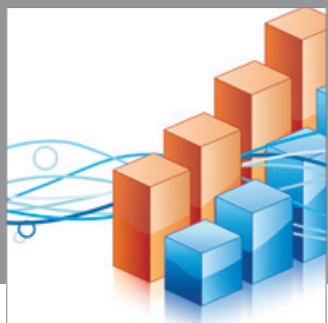

Advances in

Operations Research

mansans

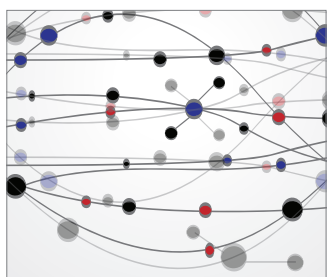

The Scientific World Journal
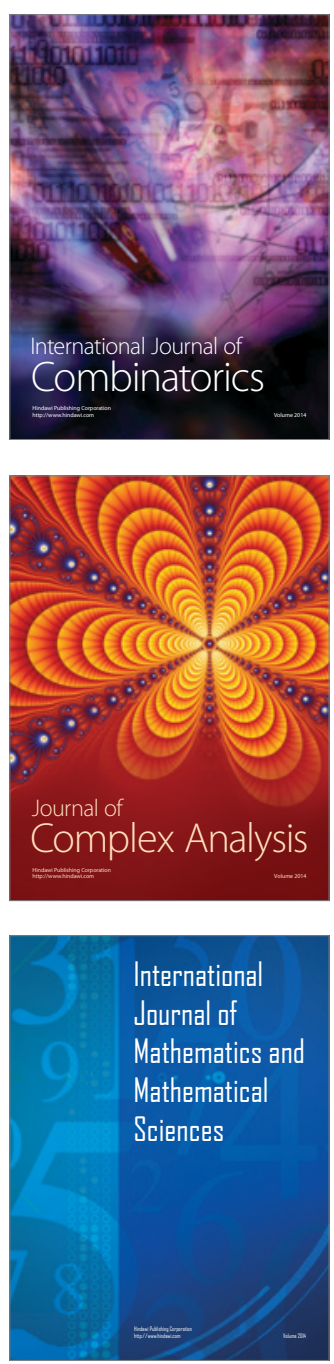
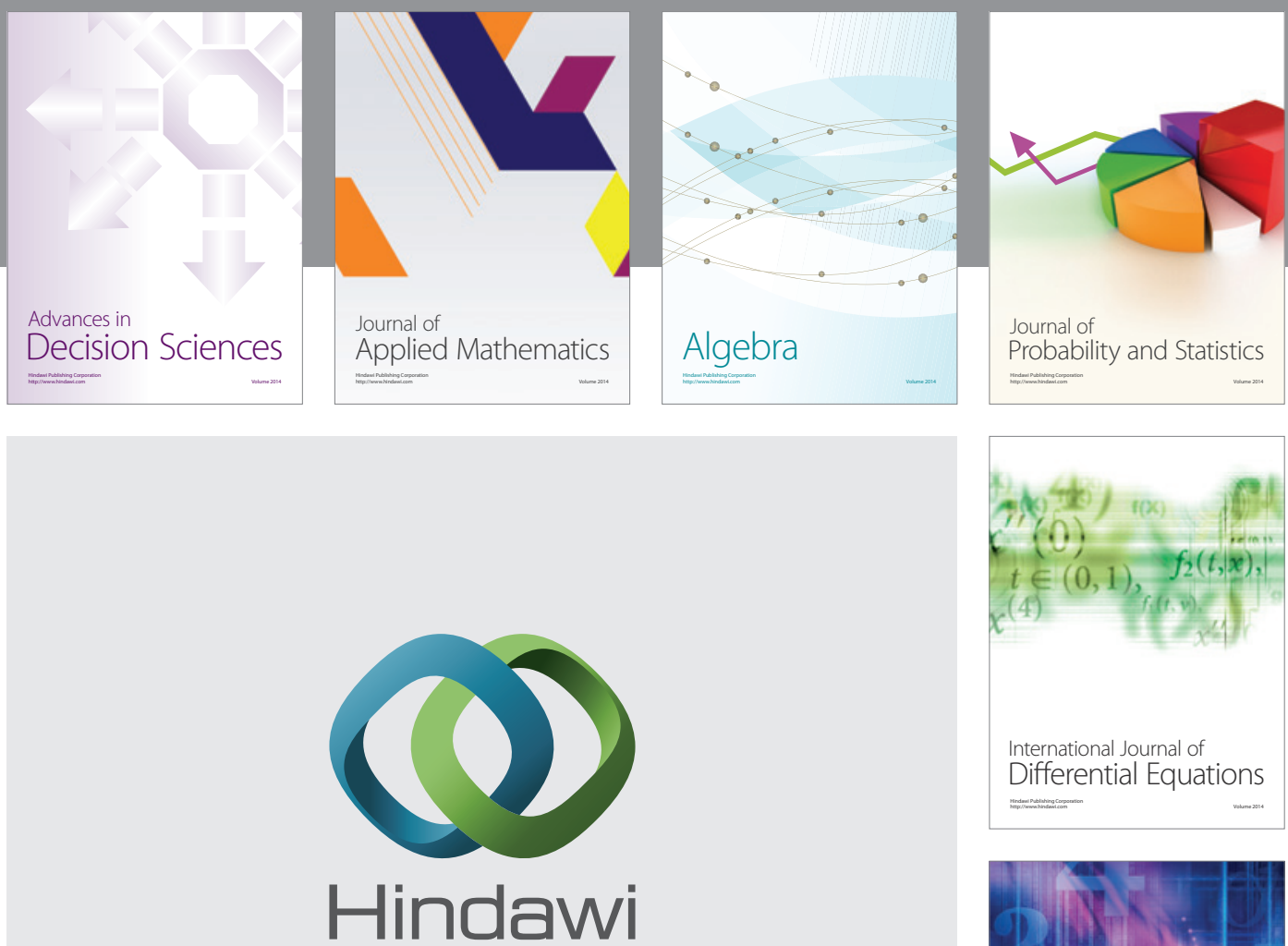

Submit your manuscripts at http://www.hindawi.com
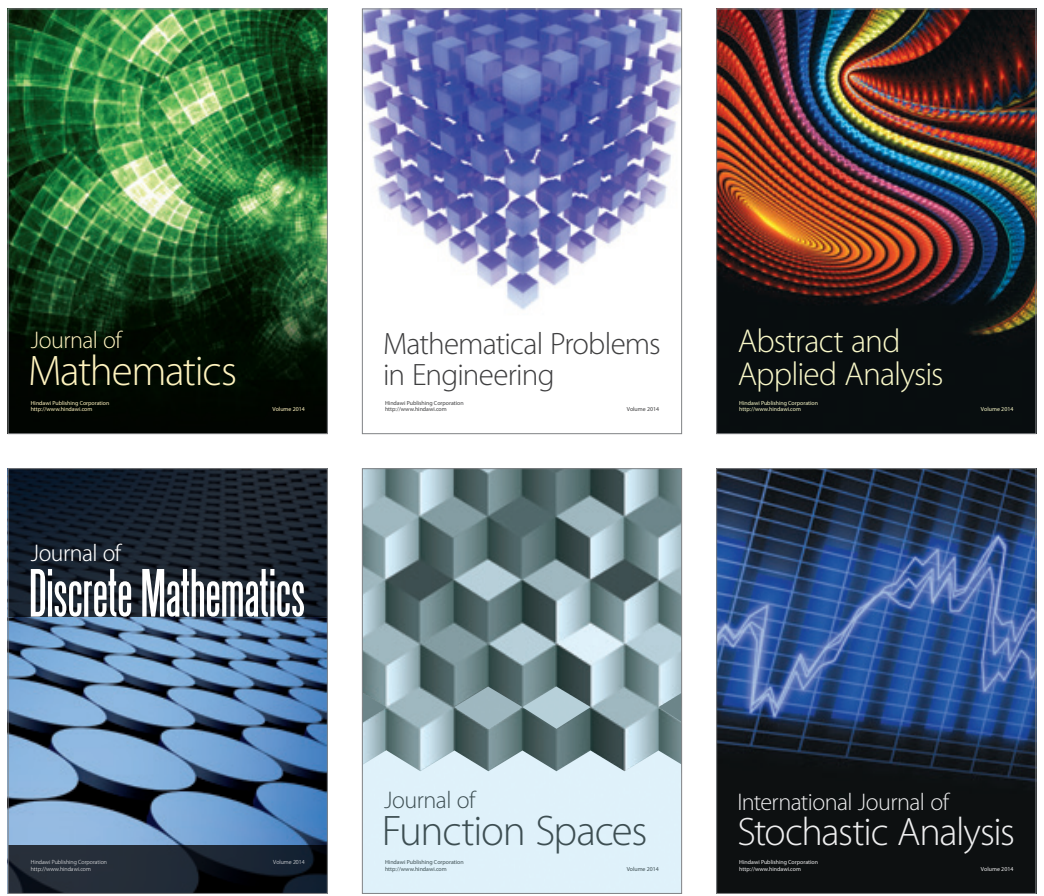

Journal of

Function Spaces

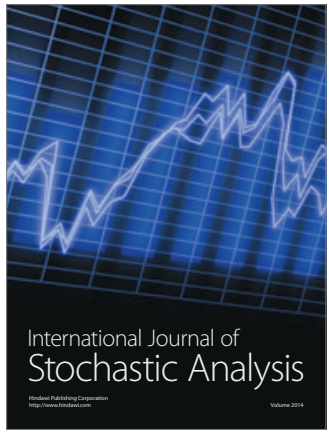

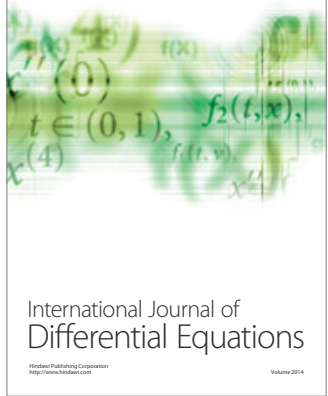
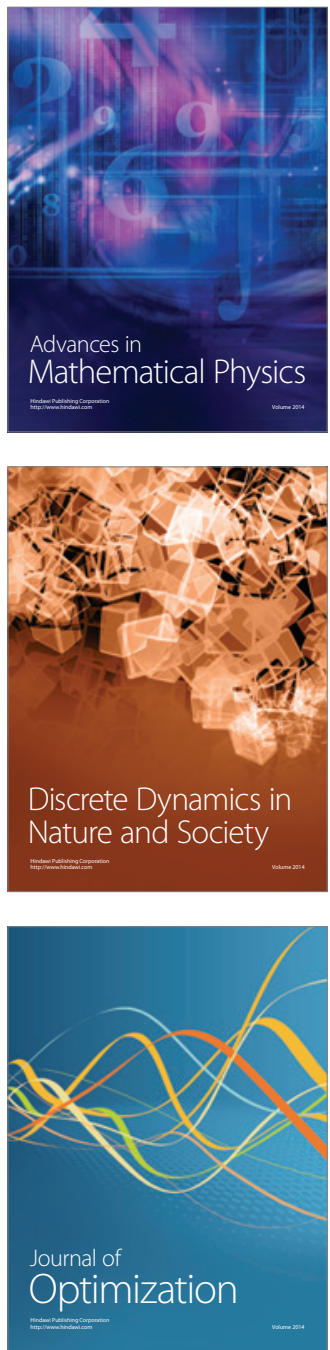